\title{
2010 Workshop: Mucosal Immunity in the Male and Female Reproductive Tract and Prevention of HIV Transmission
}

\author{
Charles R. Wira ${ }^{1}$ Fulvia Veronese ${ }^{2}$
}

E DITOR: In June 2010, a workshop entitled "Mucosal Immunity in the Male and Female Reproductive Tract and Prevention of HIV Transmission" was organized by the National Institute of Allergy and Infectious Diseases (NIAID) of the National Institutes of Health (NIH) in collaboration with the Dartmouth Medical School at the Dartmouth Hitchcock Medical Center.

This meeting was a first of its kind since it brought together leading scientists from around the world who are actively working in the area of heterosexual transmission of HIV, HIV mucosal immunity, and reproductive immunology. In a workshop/presentation format, researchers discussed HIV transmission and immune responses in the male and female reproductive tracts (RTs) and explored ways to harness this knowledge for the development of novel and more comprehensive approaches to prevention. Despite the fact that HIV is mainly transmitted sexually through the genital tract, the immune systems of the male and female genital mucosa and the ways in which they are influenced by hormonal balance have received very little attention. Compared with other mucosae, the male and female RTs are covered by distinct epithelial cell layers and types of mucus, have different commensal flora, and have unique innate and adaptive immune mechanisms. The meeting at Dartmouth aimed to fill this gap by discussing the most recent developments in basic, clinical, and translational studies in the field of reproductive immunology as they relate to the transmission of HIV, highlighting the potential of immunity in the male and female RTs in preventing HIV infection, and facilitating cross-fertilization between HIV researchers and those in the field of reproductive immunology.

The organizing committee composed of Drs. Charles R. Wira, Dartmouth Medical School; Fulvia Veronese, NIAID; Geetha Bansal, NIAID; Susan Cu-Uvin, the Warren Alpert Medical School of Brown University; Charu Kaushic, McMaster University; Jiri Mestecky, University of Alabama at Birmingham; and Robin Shattock, St. Georges, University of London, developed a scientific agenda that focused on a broad range of cross-cutting topics to be addressed by leaders in the field. Topics included the unique characteristics of the immune system in the female and male RTs, which have relevance to HIV transmission, and early virological and immunological events in male-to-female and female-to-male transmission of HIV. Functional and immunological distinctions between the gastrointestinal and RTs were highlighted and contrasted to male and female RT biology and HIV transmission. Lessons learned from reproductive immunology were discussed, and the unique relationship between factors that influence sexual HIV transmission and human reproductive biology were brought together to identify novel approaches to prevent HIV transmission and to measure genital immune responses to HIV. Lively discussions resulted in new understandings of the complexities of the male and female RTs as they pertain to susceptibility to infection and protection against HIV, and highlighted the gaps that remain to be answered.

The scientific meeting was followed by a public session entitled "The Forgotten Epidemic: AIDS in the 21st Century" for the at-large scientific faculty at Dartmouth and the general public, which focused on the impact HIV / AIDS is still having and the challenges we are confronting for its prevention and treatment. The public session was well-attended and highlighted the importance of the prevention and treatment of AIDS to the general public in juxtaposition to our scientific understanding of the processes involved in HIV transmission, treatment, and prevention.

Given the groundbreaking nature of this meeting, the American Journal of Reproductive Immunology is publishing on March 1, 2011, a special issue dedicated exclusively to the immunology of the female and male RTs and HIV sexual transmission and its prevention. This special issue includes review articles written by invited speakers and two letters to the editors by Dr. C. Everett Koop, former Surgeon General of the United States, who delivered the keynote address; and Ms. Dawn Averitt Bridge, founder of The Well Project; who spoke about the impact that HIV / AIDS is still having on different populations of women in the United States.

\section{Author Disclosure Statement}

No competing financial interests exist.

\author{
Address correspondence to: \\ Fulvia Veronese \\ Division of AIDS, NIAID, NIH, DHHS \\ 6700 Rockledge Drive, Room 5122 \\ Bethesda, Maryland 20892 \\ E-mail: veronesf@niaid.nih.gov
}

\footnotetext{
${ }^{1}$ Dartmouth Medical School, Lebanon, New Hampshire.

${ }^{2}$ Division of AIDS, NIAID, NIH, DHHS, Bethesda, Maryland.
} 
